\title{
Effects on hearing and tinnitus following Dupilumab treatment of severe asthma with chronic rhinosinusitis -
} a case report*

\author{
Mads Thorsberger ${ }^{1}$, Celeste Porsbjerg², Jesper Yde ${ }^{3}$, Kasper Aanæs ${ }^{4}$ \\ ' Dept. of Otorhinolaryngology, Head and Neck Surgery, Copenhagen university hospital, Rigshospitalet, Copenhagen, \\ Denmark \\ ${ }^{2}$ Dept. of Respiratory Medicine, Copenhagen university hospital, Bispebjerg, Copenhagen, Denmark, \\ ${ }^{3}$ Dept. of Audiology, Copenhagen university hospital, Bispebjerg, Copenhagen, Denmark, \\ ${ }^{4}$ Dept. of Otorhinolaryngology, Head and Neck Surgery, Copenhagen university hospital, Rigshospitalet, Copenhagen, \\ Denmark
}

Rhinology Online, Vol 4: 73 - 76, 2021 http://doi.org/10.4193/RHINOL/21.002

\begin{abstract}
Background: Dupilumab (anti-IL-4R) is a novel biological agent approved for treatment of severe asthma with chronic rhinosinusitis. Beneficial effects on hearing have only been sparsely decribed.

Case presentation: In this case story we present a 48-year woman, who experienced remarkably improved hearing and reduction of tinnitus after initiation of Dupilumab. Progress was monitored by respiratory physicians in collaboration with rhinologists and audiologists. SNOT-22 score improved from 83 to 27 and audiograms obtained before and during treatment illustrate a reduced air-bone gap and an improvement of speech recognition threshold from $25 \mathrm{~dB}$ (both ears) to 15 and 10 (left and right ear respectively).
\end{abstract}

Conclusions: This case story implies a beneficial effect of Dupilumab treatment in patients with united airway disease with hearing impairment. The authors suggest an additional otological perspective in addition to standard monitoring.

Key words: Dupilumab, anti-IL-4R, hearing, eosinophilic otitis media, chronic rhinosinusitis

\section{Introduction}

Anti-lgE, anti-IL-5 and anti-IL-4R are established biologic treatments for severe asthma. Dupilumab (anti-IL-4R) was approved for treatment of recalcitrant chronic rhinosinusitis (CRS) with polyps in 2019. Criteria for biologic treatment comprise: prior endoscopic sinus surgery (ESS) and three of following five: type 2 inflammation, need for systemic corticosteroids, impaired quality of life (SNOT-22 score), loss of smell and comorbid asthma. In the European Position Paper on Rhinosinuitis and Polyps (EPOS) $2020^{\text {(1) }}$ otitis media is sparsely mentioned and only in relation to paediatric or secondary CRS. However, two questions in the SNOT-22 questionnaire ask about ear symptoms: "ear fullness" and "ear pain" respectively, knowing that CRS-patients have an increased risk of ear problems ${ }^{(2)}$.

Eosinophilic otitis media (EOM) is a disease entity characterized by the presence of a highly viscous yellowish effusion containing eosinophils; immunoglobulin $\mathrm{E}(\mathrm{IgE})$ and eosinophil chemoattractants ${ }^{(3)}$. In 2011, diagnostic criteria for EOM were proposed and have since been adopted worldwide ${ }^{(4)}$. These criteria include association with bronchial asthma and nasal polyposis, and resistance to conventional treatment for otitis media. In 190 patients with EOM approximately 50\% showed deterioration in bone conduction threshold and $6 \%$ became deaf ${ }^{(5)}$. Standard treatment consists of mesotympanic steroid, although anti-IL-5 and anti-IgE has been successfully used in treatment of non-granulomatous $\mathrm{EOM}^{(6,7)}$. The pathogenesis 

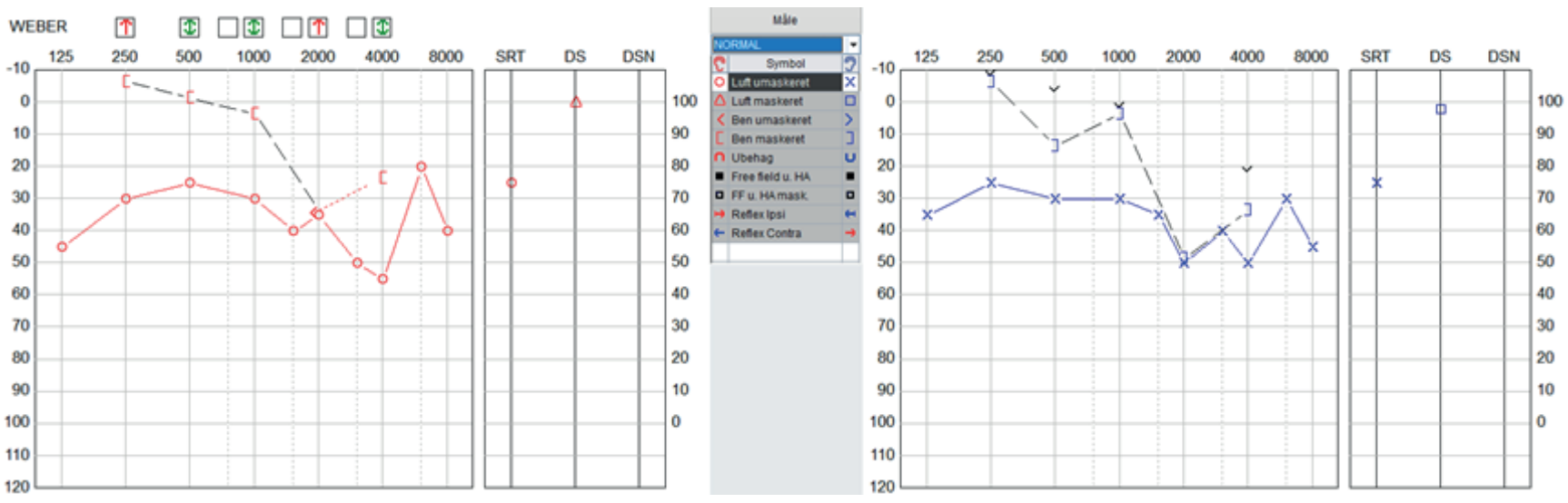

Figure 1. Hearing test two weeks prior to initiation of Dupilumab. X-axis: sound level in decibel, Y-axis: Frequency in Hertz. SRT: Speech Recognition Threshold, DS: Discrimination Score o-o: air conduction right ear, $\mathrm{x}-\mathrm{x}$ : air conduction left ear.

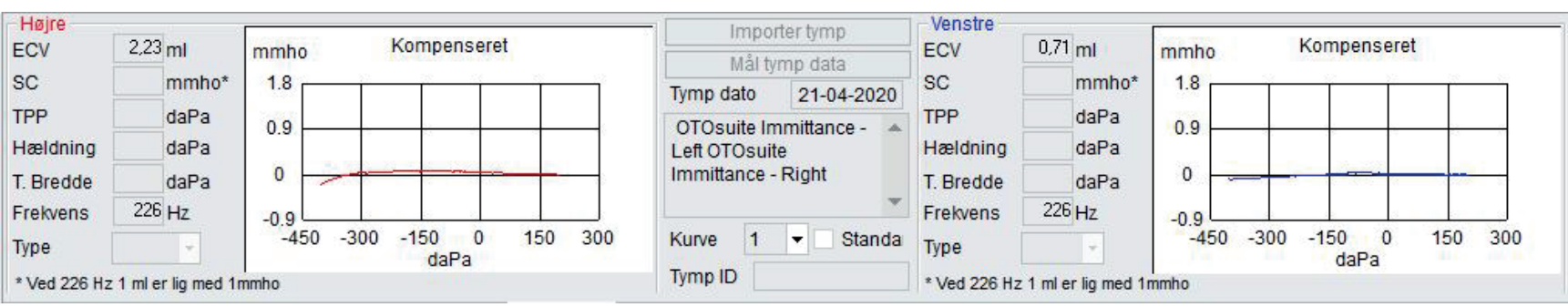

Figure 2. Tympanogram prior to initiation of Dupilumab. Type B curves with relatively small ear canal volumes $(E C V)$ indicating fluid filled middle ears are seen on both right and left side (red and blue respectively).

of EOM is still open to investigation, though a case series study conducted by Matsubara et al. ${ }^{(8)}$ supports the hypothesis of eosinophil migration via the Eustachian tube to the middle ear. In patients with EOM, high IgE levels have been demonstrated in effusion compared with serum level, and many lgE-immunopositive cells were found in the middle ear mucosa.

\section{Case presentation}

An otherwise healthy 48-year-old woman known with N-ERD (NSAID-exacerbated Respiratory Disease) had over the last decade had endoscopic sinus surgery (ESS) 12 times. The latest ESS took place in November 2019 due to a SNOT-22 score of 83 (range 0-110) in spite of appropriate medical therapy. The clinical effect lasted for less than four months, after which she again was bothered by postnasal drip, loss of smell and decreased hearing with left sided tinnitus. She had bilaterally thick secretions and nasal oedema, but only bilateral polyps grade $1-2$, not obstructing the nasal flow or the Eustachian tube. The patient had an otherwise unremarkable medical history but had over the last few years had one or two episodes of acute otitis media. Standard grommets were inserted by the patient's own ear, nose and throat specialist thrice on each ear. The patient described that thick mucus was found in the middle ear. Last grommet placement was approximately 3 months prior to Dupilumab initiation. The patients reported temporary hearing improvement after each procedure, but the effect faded as the tubes were clotted or expelled. An MRI of fossa posterior showed signs of bilateral maxillary sinusitis and sphenoiditis as well as opacification in the right mastoid cells.

Due to "late onset severe eosinophilic asthma" and her CRS symptoms, we initiated treatment with Dupilumab in March 2020.

At the time of treatment initiation, her lung function was normal, with an FEV1 of $89 \%$ of predicted, as was her exhaled nitric oxide (FeNO) of 12 ppb-a marker of eosinophilic airway inflammation, and she had not had any asthma exacerbations for the past year. She did however have significant upper airway symptoms, with a SNOT score of 83.

After three months of treatment, her SNOT-22 score dropped significantly from 83 to 27 . The patient reported tremendous improvement of her symptoms, including significantly improved hearing and a reduced tinnitus. No side effects were reported. The hearing status was monitored at monthly intervals. All hearing tests were performed in a double walled sound insulated audiometric booth, using calibrated audiometric equipment. The initial audiometry showed a mixed hearing loss, consisting of a 30-40 dB bilateral conductive hearing loss combined with a mild sensorineural hearing loss in the higher frequencies and speech recognition threshold at $25 \mathrm{~dB}$ on both ears (Figure 1). Otomicroscopy showed grommets in place, but the patient had 

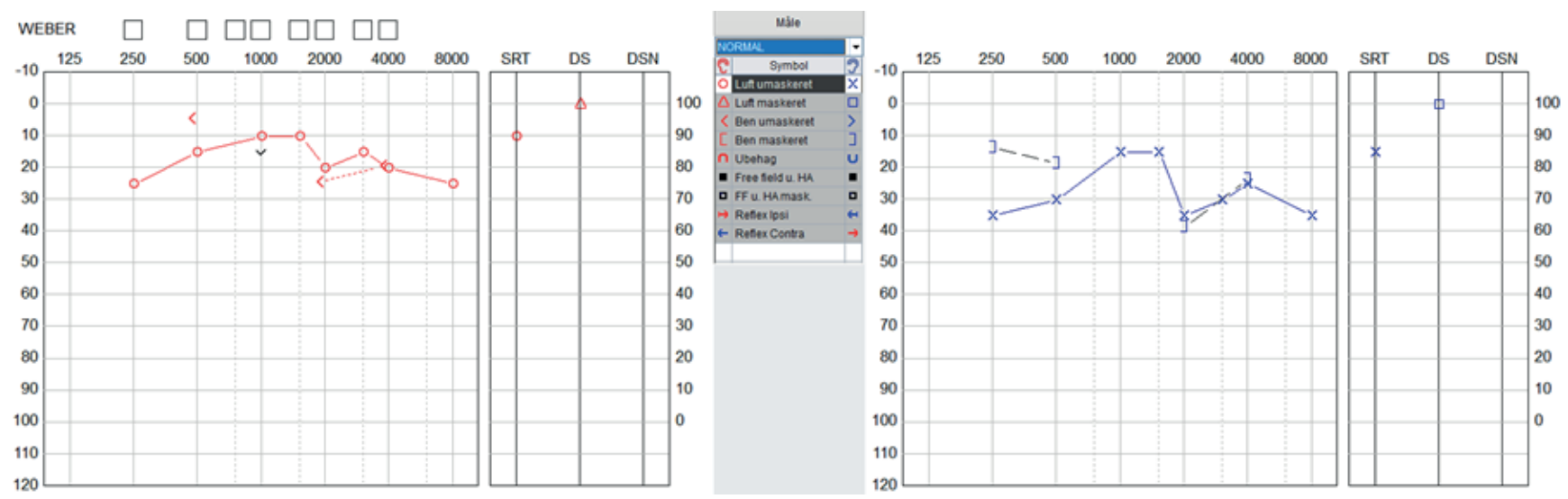

Figure 3. Hearing test after 3 months of treatment with Dupilumab. X-axis: sound level in decibel, Y-axis: frequency in Hertz. SRT: Speech Recognition Threshold, DS: Discrimination Score o-o: air conduction right ear, $x-x$ : air conduction left ear.

\begin{tabular}{|l|r|}
\hline ECV & 4,49 \\
\hline TPP & \\
\hline Hældning & \\
\hline T. Bredde & \\
\hline Frekvens & \\
\hline Type & \\
\hline
\end{tabular}
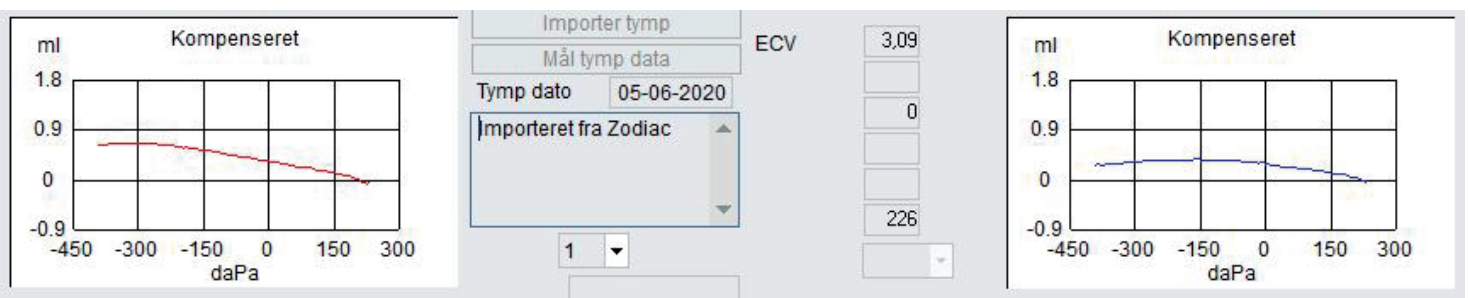

Figure 4. Tympanogram during Dupilumab treatment. Type B curves are still present on both sides, but ear canal volumes (ECV) are now large indicating functioning ventilation tubes and air-filled middle ears on both right and left side (red and blue respectively).

a Type B tympanogram with normal volumes indicating tube obstruction (Figure 2).

Follow up audiometry shows a decrease in the overall air-bone gap, most noticeable on the right ear (Figure 3). Speech recognition threshold level improved to 10 and $15 \mathrm{~dB}$ (left and right ear respectively). The dip in the bone conduction thresholds around $2 \mathrm{kHz}$ probably represents Carhart's phenomenon. The grommets were still in place and the type B tympanogram now showed large volumes indication functioning tubes (Figure 4).

\section{Discussion}

To our knowledge, only one other recent case study reports improved hearing after treatment with Dupilumab ${ }^{(9)}$. In our patient report, improvement was shown objectively by an audiogram, but especially self-reported. This patient is a good example of a patient having global airway disease. Her asthma worsens due to upper airways symptoms. In general, the treatment with Dupilumab show promising results in asthma and CRS patients within few weeks ${ }^{(10)}$. However, little attention has been on middle ear problems in CRS patients.

Ear fullness, ear pain, and decreased hearing can be caused by large polyps obstructing the Eustachian tube and choana, thus creating a negative pressure in the middle ear. However, this was not the case in this patient since nasal endoscopy prior to Dupilumab did not find obstructive nasal polyposis. We hypothesize that the patient suffers from eosinophilic otitis media, which improved due to the biologic treatment. Though the eosinophilic ear in the recent years has been given increasing attention, literature is still sparse and little attention has been given to hearing in the treatment with biologic agents. As IgE levels in middle ear effusion has been shown to correlate with hearing, we speculate that Dupilumab as an anti-IL-4R agent may reduce this $\mathrm{IgE}$ concentration and thereby improve hearing. Since hearing loss worsens over time, initiation of adequate treatment as early as possible is essential to the patient's quality of life. Insertion of ventilation tubes in this patient category is debatable. While some may experience a relief with improved hearing and reduced fullness, the effect is often temporary and the possibility of chronic perforation with secretion as well as the risk involved with repeated anaesthesia must be considered.

Systematic biological treatment for chronic rhinosinusitis is about to become available. Similar to the international severe asthma registries, we find it crucial to follow these patients closely creating a database with data on sinonasal symptoms and findings, asthma symptoms and measurements, medication, allergies, eosinophilic counts and side-effects. In this database, we will now include a tympanometry and a hearing test in all patients reporting of decreased hearing. 


\section{Conclusions}

The present case illustrates that severe eosinophilic asthma is a systemic disease, which may affect the ears and induce hearing impairment, as well as the well-known association with CRSwNP. Hence, we advocate that hearing is routinely assessed through a thorough history, as well as hearing tests in patients with complaints of hearing impairment.

Finally, assessment of hearing problems should be included in clinical registries on patients with severe asthma and/or CRSwNP, to enable us to understand how common this problem is, and possible support hearing impairment to become part of the future set of indications for biological treatments for severe Type 2 disease.

\section{Authorship contribution}

$\mathrm{CP}$ conducted and interpreted the respiratory examinations. KA conducted and interpreted the rhinology examinations. JY was in charge of the audiology interpretations. MT wrote the manuscript. All authors contributed and accepted the final manuscript.

\section{Acknowledgments}

Not applicable.

\section{Funding}

None

\section{Ethics approval and consent to participate}

Not applicable.

\section{Consent for publication}

A written consent for publication of this case story was obtained before preparation of the manuscript.

\section{Availability of data and materials Not applicable.}

\section{Conflict of interest}

Celeste Porsbjerg: prior member of Sanofi advisory board; Kasper Aanæs: prior member of Sanofi advisory board; Jesper Yde: None; Mads Thorsberger: None

\section{References}

1. Fokkens WJ, Lund VJ, Hopkins C, et al. European Position Paper on Rhinosinusitis and Nasal Polyps. Rhinology. 2020 Feb 20;58(Suppl S29):1-464

2. Hong SN, Lee WH, Lee SH, Rhee CS, Lee $\mathrm{CH}$, Kim JW. Chronic rhinosinusitis with nasal polyps is associated with chronic otitis media in the elderly. Eur Arch Otorhinolaryngol. 2017 Mar;274(3):14631470

3. lino Y. Eosinophilic otitis media: a new middle ear disease entity. Curr Allergy Asthma Rep. 2008 Nov:8(6):525-30.

4. lino $Y$, Tomioka-Matsutani S, Matsubara A, Nakagawa T, Nonaka M. Diagnostic criteria of eosinophilic otitis media, a newly recognized middle ear disease. Auris Nasus Larynx. 2011 Aug;38(4):456-61.

5. Suzuki H, Matsutani S, Kawase T. Epidemiologic Surveillance of 'Eosinophilic Otitis Media' in Japan. J Stage. 2004, 14, 2, 112-117

6. lino Y, Takahashi E, Ida S, Kikuchi S. Clinical efficacy of anti-IL-5 monoclonal antibody mepolizumab in the treatment of eosinophilic otitis media. Auris Nasus Larynx. 2019 Apr:46(2):196-203.

7. Iino $Y$, Hara M, Hasegawa $M$, et al. Clinical efficacy of anti-IgE therapy for eosinophilic otitis media. Otol Neurotol. 2012 Sep;33(7):1218-24.

8. Ohta N, Ueki S, Konno Y, et al. ETosisderived DNA trap production in middle ear effusion is a common feature of eosinophilic otitis media. Allergol Int. 2018 Jul;67(3):414-416

9. lino $Y$, Sekine $Y$, Yoshida S, Kikuchi S Dupilumab therapy for patients with refractory eosinophilic otitis media associated with bronchial asthma. Auris Nasus Larynx. 2020 Sep 14;S0385-8146(20)30248-0.

10. Bachert C, Han JK, Desrosiers M, et al. Efficacy and safety of dupilumab in patients with severe chronic rhinosinusitis with nasal polyps (LIBERTY NP SINUS-24 and LIBERTY NP SINUS-52): results from two multicentre, randomised, double-blind, placebo-con- trolled, parallel-group phase 3 trials. Lancet. 2019 Nov 2;394(10209):1638-1650.

Mads Thorsberger

Dept. of Otorhinolaryngology

Head and Neck Surgery

Copenhagen University Hospital

Rigshospitalet

Copenhagen

Denmark

Tel: +4522385092

E-mail:

mads.thorsberger@regionh.dk

ISSN: 2589-5613 / @2021 The Author(s). This work is licensed under a Creative Commons Attribution 4.0 International License. The images or other third party material in this article are included in the article's Creative Commons license, unless indicated otherwise in the credit line; if the material is not included under the Creative Commons license, users will need to obtain permission from the license holder to reproduce the material. To view a copy of this license, visit http://creativecommons.org/licenses/by/4.0/ 